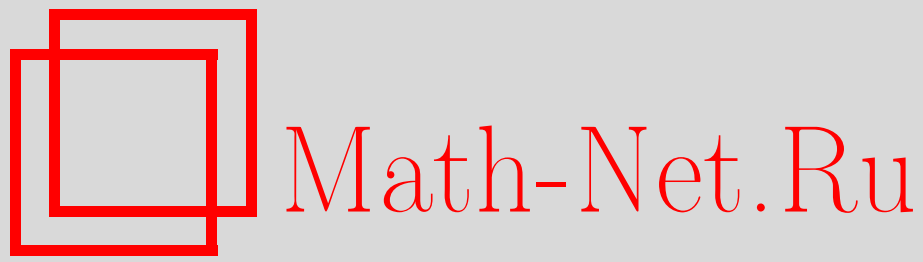

В. П. Маслов, Фазовые переходы в вероятностной игре, Матем. заметки, 2003, том 73, выпуск 4, 637-640

DOI: https://doi.org/10.4213/mzm2668

Использование Общероссийского математического портала MathNet.Ru подразумевает, что вы прочитали и согласны с пользовательским соглашением http://www . mathnet.ru/rus/agreement

Параметры загрузки:

IP: 18.234 .197 .8

26 апреля 2023 г., 17:51:08

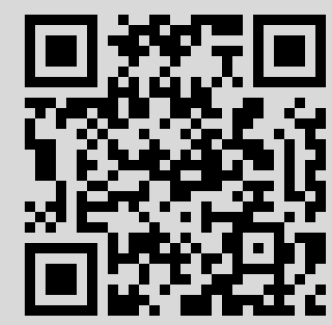




\section{ФАЗОВЫЕ ПЕРЕХОДЫ В ВЕРОЯТНОСТНОЙ ИГРЕ}

\section{В.П. Маслов}

В предыдущей работе [1] было показано, как чисто вероятностные задачи можно перевести на язык квантовой механики: введением оператора Гамильтона $\widehat{H}$ в гильбертовом пространстве $l_{2}$ (в конечномерном случае), и что частотную вероятность уровня энергии $\varepsilon_{n}$ оператора Гамильтона $\widehat{H}$ можно интерпретировать как его кратность $k\left(\varepsilon_{n}\right)$. Автором было введено понятие оператора свободной энергии $\widehat{F}$ как оператора с собственными значениями $\left(\varepsilon_{n}-\theta \ln k\left(\varepsilon_{n}\right)\right)$ и теми же собственными функциями $[2$, с. 65 , формулы 5,6$]$. Рассматривается модель кристалла с условиями Борна-Кармана с $M$ ячейками, между которыми потенциальные барьеры велики и расстояние между энергиями одной зоны (собственные функции симметричны) столь малы, что ими можно пренебреч. Тогда кратность каждого собственного значения умножается на $M$. Рассматриваются (как обычно) две зоны $\varepsilon_{1}$ и $\varepsilon_{2}$ (двухуровневая модель) со следуюшим парным взаимодействием. Любые два бозона, находящиеся на верхнем уровне, отдают квант энергии, обратно пропорщиональный числу частиц $N$. При некоторых условиях на разность $\left(\varepsilon_{1}-\varepsilon_{2}\right)$ и потенциал $V$ имеется одно метастабильное состояние, интерпретируемое как сверхтекучее [3]. Можно показать, что для собственных значений оператора свободной энергии, отвечающих этому метастабильному состоянию, зависяших от $\theta$, при некотором $\theta$ происходит фазовый переход второго рода с критическим значением $1 / 2$. Эту задачу мы переформулируем здесь как некоторую вероятностную игру.

Рассмотрим следующую игру. Одновременно кидают $N$ одинаковых костей, каждая кость имеет $M \gg 1$ граней, грани с номером $i$ соответствует число $\lambda_{i}, \lambda_{i}<\lambda_{i+1}$. Выигрыш (доход) вычисляется следуюшим образом. Обозначим через $n_{i}$ число костей, у которых выпала грань с номером $i$. Очевидно, что $\sum_{i=1}^{M} n_{i}=N$. Пусть $G_{1}, G_{2}>0$ - целые числа такие, что $G_{1}+G_{2}=M, \lambda_{i}<0$ при $i \leqslant G_{1}, \lambda_{j}>0$ при $j<G_{1}$. Введем для броска одной кости осредненный проигрыш

$$
\omega_{1}=\frac{1}{G_{1}} \sum_{i=1}^{G_{1}} \lambda_{i}
$$

и выигрыш

$$
\omega_{2}=\frac{1}{G_{2}} \sum_{i=1}^{G_{2}} \lambda_{G_{1}+i}
$$

тогда выигрыш для броска $N$ костей задается формулой

$$
\mathscr{E}=\omega_{1} N_{1}+\omega_{2} N_{2},
$$

где $N_{1}, N_{2}$ выражаются следуюшим образом:

$$
N_{1}=\sum_{i=1}^{G_{1}} n_{i}, \quad N_{2}=\sum_{i=1}^{G_{2}} n_{i+G_{1}} .
$$

Очевидно, что $N_{1}+N_{2}=N$. Будем считать, что кости являются неразличимыми. В таком случае число возможных вариантов бросания $N$ костей с выигрышем $\mathscr{E}=\omega_{1} N_{1}+\omega_{2} N_{2}$ равно

$$
\Gamma\left(N_{1}, N_{2}\right)=\sum_{K_{1}=0}^{N} \cdots \sum_{K_{M}=0}^{N} \delta_{L_{1} N_{1}} \delta_{L_{2} N_{2}},
$$

где $\delta_{L N}$ - символ Кронекера, а $L_{1}, L_{2}$ зависят от $K_{1}, \ldots, K_{M}$ следуюшим образом:

$$
L_{1}=\sum_{i=1}^{G_{1}} K_{i}, \quad L_{2}=\sum_{i=1}^{G_{2}} K_{i+G_{1}} .
$$


Легко убедиться, что (5) может быть записано в виде

$$
\Gamma\left(N_{1}, N_{2}\right)=\frac{\left(N_{1}+G_{1}-1\right) !}{N_{1} !\left(G_{1}-1\right) !} \cdot \frac{\left(N_{2}+G_{2}-1\right) !}{N_{2} !\left(G_{2}-1\right) !} .
$$

Введем энтропию, отвечающую выигрышу $\mathscr{E}=\omega_{1} N_{1}+\omega_{2} N_{2}$, следующим образом:

$$
S\left(N_{1}, N_{2}\right)=\ln \left(\Gamma\left(N_{1}, N_{2}\right)\right) \text {. }
$$

Кроме того, будем считать, что в игре взимается налог на выигрыш. А именно, у каждой пары выигравших костей - таких костей, у которых выпала грань с номером $i>G_{1}$ - отнимается сумма $V / N$. Поскольку таких пар всего $N_{2}\left(N_{2}-1\right) / 2$ штук, окончательный выигрыш (доход) в игре составит

$$
\mathscr{E}\left(N_{1}, N_{2}\right)=\omega_{1} N_{1}+\omega_{2} N_{2}-\frac{V N_{2}\left(N_{2}-1\right)}{2 N} .
$$

Рассмотрим случай $N \gg 1$ в описанной игре. Обозначим $G_{1} / N=g_{1}, G_{2} / N=g_{2}$. Легко убедиться, что в этом случае

$$
\begin{aligned}
\frac{\mathscr{E}\left(N_{1}, N_{2}\right)}{N} \simeq & \mathscr{E}(n)=\omega_{1}+\omega \cdot n-\frac{V n^{2}}{2} \\
\frac{S\left(N_{1}, N_{2}\right)}{N} \simeq & S(n)=\left(n+g_{2}\right) \ln \left(1+\frac{n}{g_{2}}\right)-n \ln \left(\frac{n}{g_{2}}\right) \\
& +\left(1-n+g_{1}\right) \ln \left(1+\frac{1-n}{g_{1}}\right)-(1-n) \ln \left(\frac{1-n}{g_{1}}\right)
\end{aligned}
$$

где $n=N_{2} / N, \omega=\omega_{2}-\omega_{1}>0$. Рассмотрим задачу о нахождении экстремальных значений выигрыша (9) при фиксированной энтропии (10). Такая задача решается с помошью метода Лагранжа. Функция Лагранжа имеет вид

$$
\begin{aligned}
f(n, \theta)= & \omega_{1}+\omega n-\frac{V n^{2}}{2}+\theta\left(n \ln \left(\frac{n}{g_{2}}\right)-\left(g_{2}+n\right) \ln \left(1+\frac{n}{g_{2}}\right)\right. \\
& \left.+(1-n) \ln \left(\frac{1-n}{g_{1}}\right)-\left(g_{1}+1-n\right) \ln \left(1+\frac{1-n}{g_{1}}\right)\right)
\end{aligned}
$$

где $\theta$ - множитель Лагранжа, который будем называть волатильностью, $n \in[0,1]$. Далее будем считать, что задана волатильность, а энтропию будем выражать через нее. По определению имеем $\omega>0$. Будем также считать далее, что

$$
V>\omega, \quad \theta>0 .
$$

Рассмотрим задачу о нахождении минимума функции (11) при фиксированных $\omega, V, g_{1}, g_{2}$.

Поскольку $\theta>0$ и $f(n, \theta)$ является бесконечно дифференцируемой функцией переменной $n$, экстремальное значение $f(n, \theta)$ достигается при $n=n_{0}\left(\theta, V, \omega, g_{1}, g_{2}\right) \in(0,1)$, где $n_{0}\left(\theta, V, \omega, g_{1}, g_{2}\right)$ удовлетворяет уравнению

$$
0=\frac{\partial f}{\partial n}\left(n_{0}, \theta\right)=\omega-V n_{0}+\theta \ln \left(\frac{n_{0}}{g_{2}+n_{0}} \cdot \frac{g_{1}+1-n_{0}}{1-n_{0}}\right) .
$$

Уравнение (13) имеет, по крайней мере, одно решение, так как

$$
\frac{\partial f}{\partial n} \rightarrow+\infty \text { при } n \rightarrow 1, \quad \frac{\partial f}{\partial n} \rightarrow-\infty \text { при } n \rightarrow 0 .
$$

Кроме того, в силу

$$
\frac{\partial^{4} f}{\partial n^{4}}\left(n_{0}, \theta\right)=2 \theta\left(\frac{1}{n^{3}}-\frac{1}{\left(g_{2}+n\right)^{3}}+\frac{1}{(1-n)^{3}}-\frac{1}{\left(g_{1}+1-n\right)^{3}}\right)>0
$$

уравнение (13) не мох:ет иметь более трех решений. 
Если выполнены условия (12), то легко показать, что при фиксированных параметрах $\omega, V, g_{1}$, $g_{2}$ уравнение (13) имеет три решения при малых значениях волатильности $\theta$ и одно решение при больших значениях волатильности. Асимптотика решений при $\theta \rightarrow 0$ есть

$$
\begin{gathered}
n_{1} \sim \frac{g_{2}}{1+g_{1}} \exp \left(-\frac{\omega}{\theta}\right), \quad n_{2}-1 \sim-\frac{g_{1}}{1+g_{2}} \exp \left(-\frac{V-\omega}{\theta}\right), \\
n_{3}-\frac{\omega}{V} \sim \frac{\theta}{V} \ln \left(\frac{\omega / V}{g_{2}+\omega / V} \cdot \frac{g_{1}+1-\omega / V}{1-\omega / V}\right) .
\end{gathered}
$$

При $\theta \rightarrow \infty$ асимптотика единственного решения уравнения (13) есть

$$
n_{0}-\frac{g_{2}}{g_{1}+g_{2}} \sim \frac{1}{\theta}\left(V \frac{g_{2}}{g_{1}+g_{2}}-\omega\right) \frac{g_{1} g_{2}\left(1+g_{1}+g_{2}\right)}{\left(g_{1}+g_{2}\right)^{3}} .
$$

Таким образом, видим, что при фиксированных значениях параметров $V, \omega, g_{1}, g_{2}$ в игре возможен фазовый переход при изменении волатильности $\theta$. Далее будут исследованы фазовые переходы, вызванные изменением налога. Вообще, в зависимости от значений $\theta, V, \omega, g_{1}, g_{2}$ уравнение (13) может иметь три решения (в таком случае функция (11) имеет локальный и глобальный минимумы и максимум на интервале $n \in(0,1))$ или меньшее число решений (тогда функция (11) имеет один глобальный минимум на интервале $n \in(0,1))$. Значения параметров $\theta, V, \omega, g_{1}, g_{2}$, при которых уравнение (13) имеет два решения, являются критическими; при приближении к этим значениям со стороны, где существуют три решения, два решения - локальный минимум и максимум функции (11) - приближаются друг к другу и превращаются в одно при достижении критических значений параметров, а при дальнейшем изменении параметров исчезают. Критические значения параметров, при которых происходит описанная бифуркапия решений уравнения (13), определяются из уравнения

$$
0=\frac{\partial^{2} f}{\partial n^{2}}\left(n_{0}, \theta\right)=-V+\theta\left(\frac{1}{n_{0}}-\frac{1}{g_{2}+n_{0}}+\frac{1}{1-n_{0}}-\frac{1}{g_{1}+1-n_{0}}\right),
$$

где $n_{0}$ удовлетворяет уравнению (13). Покажем, что при приближении к критическим значениям производные энтропии, отвечающей решениям, испытывающим бифуркацию, стремятся к бесконечности.

Пусть параметры $\theta, \omega, g_{1}, g_{2}$ заданы. Рассмотрим фазовый переход, происходящий при изменении налога $V$. Пусть $n_{0}=n_{0}\left(\theta, \omega, g_{1}, g_{2}\right), V=V_{0}\left(\theta, \omega, g_{1}, g_{2}\right)$ удовлетворяют уравнениям $(13),(17)$. В таком случае легко получить, что асимптотика решений уравнения (13), испытывающих бифуркацию, при стремлении налога $V$ к критическому значению $V_{0}$ имеет вид

$$
n_{0}-n_{0}\left(\theta, \omega, g_{1}, g_{2}\right) \simeq \pm \sqrt{-\frac{2 \frac{\partial^{2} f}{\partial V \partial n}\left(n_{0}, \theta\right)\left(V-V_{0}\right)}{\frac{\partial^{3} f}{\partial n^{3}}\left(n_{0}, \theta\right)}}
$$

Энтропия, отвечающая решениям уравнения (13), есть

$$
S=-\frac{\partial f}{\partial \theta}\left(n_{0}, \theta\right)
$$

Ее производная по параметру $V$

$$
\frac{\partial S}{\partial V}=-\frac{\partial^{2} f}{\partial \theta \partial V}\left(n_{0}, \theta\right)-\frac{\partial^{2} f}{\partial \theta \partial n}\left(n_{0}, \theta\right) \cdot \frac{\partial n_{0}}{\partial V}=\ln \left(\frac{g_{2}+n_{0}}{n_{0}} \cdot \frac{1-n_{0}}{g_{1}+1-n_{0}}\right) \cdot \frac{\partial n_{0}}{\partial V} .
$$

В силу (18) очевидно, что при приближении налога $V$ к критическому значению $V_{0}\left(\theta, \omega, g_{1}, g_{2}\right)$ выражение (19) растет как $1 / \sqrt{\left|V-V_{0}\right|}$, т.е. имеет место фазовый переход с критическим индек$\operatorname{com} 1 / 2$. 
Заметим, что если параметры $\theta, \omega, g_{1}, g_{2}$ фиксированы, то при изменении налога $V$ могут происходить также фазовые переходы первого рода. Такие фазовые переходы возможны, когда уравнение (13) имеет три решения. В таком случае функция (11) имеет локальный и глобальный минимумы. Рассмотрим $n_{1}\left(\theta, \omega, V, g_{1}, g_{2}\right)$ и $n_{2}\left(\theta, \omega, V_{2}, g_{1}, g_{2}\right)$, непрерывно зависящие от $V$ и непрерывно дифференцируемые по $V$, решения уравнения (13). Критическое значение налога $V_{0}$ определяется из уравнения

$$
f\left(n_{1}, \theta\right)=f\left(n_{2}, \theta\right) .
$$

Если при $V<V_{0}$ точками глобального и локального минимумов функции (11) являлись соответственно $n_{1}\left(\theta, \omega, V, g_{1}, g_{2}\right)$ и $n_{2}\left(\theta, \omega, V_{2}, g_{1}, g_{2}\right)$, то после превышения налогом критического значения, при $V>V_{0}$, абсолютный минимум функции (11) достигается при $n=n_{2}\left(\theta, V, \omega, g_{1}, g_{2}\right)$, a локальный - при $n=n_{1}\left(\theta, V, \omega, g_{1}, g_{2}\right)$. Таким образом, очевидно, что при фиксированной волатильности и увеличении налога минимальный возможный выигрыш (9) в рассматриваемой игре и соответствующая ему энтропия (10) могут измениться скачком, что можно интерпретировать как финансовую катастрофу (дефолт).

В конце заметим, что при малых значениях налога, когда условие (12) $V>\omega$ не выполнено, фазовые переходы при изменении волатильности или налога невозможны. Это следует из вида функции (11) и ее производных первого (13) и более высоких порядков. Например, легко видеть, что в таком случае при малых значениях волатильности сушествует только одно решение уравнения (13), а определяемые формулами (15) $n_{2}$ и $n_{3}$ уже не являются асимптотиками каких-либо решений уравнений (13). Из формул (11), (13) следует, что если налог увеличить, то произойдет фазовый переход (кризис, дефолт, взрыв).

Автор благодарит Г. Коваля за помощь.

\section{СПИСОК ЦИТИРОВАННОЙ ЛИТЕРАТУРЫ}

1. Маслов В. П. // Матем. заметки. 2002. Т. 72. №6. С. 883-891. 2. Maslov V. P. // Russ. J. Math. Phys. 2001. V. 8. № 1. P. 55-82. 3. Боголюбов Н. Н. Избранные труды. Т. 2. Киев: Наукова думка, 1970.

Московский государственный университет им. М. В. Ломоносова

Поступило

E-mail: viktor_maslov@hotmail.com

21.02.2003 